\title{
Modeling and optimal control of fast filling process of hydrogen to fuel cell vehicle
}

\author{
Yunfeng Bai ${ }^{1}$, Caizhi Zhang ${ }^{1, *}$, Hao Duan ${ }^{1}$, Shangfeng Jiang ${ }^{2, *}$, Zhiming Zhou ${ }^{2}$, \\ Didier Grouset $^{3}$, Mingjun Zhang ${ }^{4}$, Xuefeng $\mathrm{Ye}^{5}$ \\ ${ }^{1}$ School of Automotive Engineering; The State Key Laboratory of Mechanical Transmissions; Chongqing Automotive Collaborative Innovation Centre, Chongqing \\ University, Chongqing, 400044, China \\ ${ }^{2}$ Zhengzhou Yutong Bus Co., Ltd. Yutong Industrial Park, Yutong Road, Zhengzhou, Henan Province, 450061, China \\ ${ }^{3}$ RAPSODEE, UMR CNRS 5302, IMT Mines-Albi, campus Jarlard, 81013 ALBI CT Cedex 09, France \\ ${ }^{4}$ Beijing PERIC Hydrogen Technologies Co., Ltd, Qingnian Road, Damei Center, Chaoyang District, Beijing, 100123, China \\ ${ }^{5}$ Propulsion Research Institute of Chongqing Changan New Energy Vehicle Technology Co., Ltd., Chongqing, 400000, China
}

Keywords:

Cascade storage system

Fast filling

Pressure switching coefficient

Pre-cooling temperature

Optimization algorithm

\begin{abstract}
A B S T R A C T
Due to the rapid compression of hydrogen and the Joule-Thompson effect specific to hydrogen during the fast filling process, the internal temperature of the cylinder rises sharply which may lead to hidden safety hazards. In this study, a high-pressure hydrogen filling process is considered, and a simple mathematical model of a cascade storage system of a hydrogen refilling station is developed to analyze the temperature rise in hydrogen cylinders under different working conditions. The results show the pressure switching coefficient has a great impact on the filling time, and the pre-cooling of hydrogen has a significant impact on the temperature rise and the states of charge (SOC) within cylinder. Herein, a multi-objective iterative optimization algorithm is proposed to calculate the above two controllable variables (pressure switching coefficient, pre-cooling temperature of hydrogen) with the objectives of faster refueling, lower energy consumption and higher SOC within cylinders in cascade hydrogen refueling. Besides, this method could significantly decrease energy consumption, improve SOC and allow acceptable refueling time.
\end{abstract}

\section{Introduction}

Following the fossil energy crisis, searching for renewable and clean energy has become an important issue, which may hugely affect development of human society in the future. Hydrogen energy is considered as one of the highly interesting alternative fuels in the future with the advantages of possible high efficiency, renewable production and zero-pollution [1, 2]. Hydrogen energy is being increasingly developed together with fuel cell power systems and fuel cell vehicles in recent years [3, 4]. With the gradual commercialization of fuel cell vehicles, the development of hydrogen refueling stations (HRSs) has gained more and more attention [5]. Governments around the world begin to support the construction of HRSs for their currently high capital and operating costs. Currently, high pressure hydrogen storage in refueling stations are mostly considered, as more practical, reliable, durable and so acceptable [6-8]. There are two approaches for hydrogen storage $[9,10]$. One is the buffer system requires lots of compression work for hydrogen storage. The other is cascade storage system which applied on a large scale due to its lower power consumption, appreciable when high hydrogen utilization occur.

Although the compressed hydrogen approach has advantage of technical simplicity and high filling rates[11], the fast filling speeds and the high states of charge (SOC) bring to new challenges for the on-board cylinders. The rapid increase of hydrogen temperature during the fast filling process could lead to safety hazards and so that both the filling rate and the SOC have to be reduced [12]. The maximum allowed temperature on the cylinder wall is set to $358 \mathrm{~K}$ for security [13]. Lots of experiments [14-16] and computational fluid dynamics (CFD) models have been developed to investigate the mechanism of temperature rise $[17,18]$. This rapid increase of temperature is caused by three main thermodynamic phenomena, namely compression of hydrogen, the negative Joule-Thomson effect of hydrogen and the conversion of kinetic energy into thermal energy $[19,20]$. The temperature rise within a $70 \mathrm{MPa}$ type III cylinder has been experimentally and numerically studied by Zheng et al. [14]. The effect of initial pressure, ambient

\footnotetext{
* Corresponding Authors.

E-mail addresses: czzhang@cqu.edu.cn (C. Zhang), sfjiang521@yeah.net (S. Jiang).
} 


\begin{tabular}{|c|c|c|c|}
\hline \multicolumn{2}{|c|}{ Nomenclature } & $t$ & Time (s) \\
\hline$P_{\text {swit }}$ & Target switching pressure $(\mathrm{Pa})$ & $b$ & Constant coefficient \\
\hline $\begin{array}{l}P_{\text {sto }} \\
m\end{array}$ & $\begin{array}{l}\text { Pressure of cascade storage system }(\mathrm{Pa}) \\
\text { Hydrogen mass }(\mathrm{g})\end{array}$ & $x$ & $\begin{array}{l}\text { Optimized parameters: pressure switch coefficient (PSC) } \\
\text { and pre-cooling temperature }\end{array}$ \\
\hline$\dot{m}$ & Mass flow rate $(\mathrm{g} / \mathrm{s})$ & $W(x)$ & Pre-cooling energy consumption \\
\hline & & $t(x)$ & Filling time \\
\hline $\begin{array}{l}Q \\
\rho_{g}\end{array}$ & $\begin{array}{l}\text { Heat transter rate }(W) \\
\text { Specific mass }\left(\mathrm{g} / \mathrm{m}^{3}\right)\end{array}$ & $\operatorname{SOC}(x)$ & $\begin{array}{l}\text { State of charge (ratio hydrogen mass to nominal hydrogen } \\
\text { mass within cylinder) }\end{array}$ \\
\hline$V$ & Volume $\left(\mathrm{m}^{3}\right)$ & $X$ & Constraint condition of optimization algorithm \\
\hline$T$ & Temperature (K) & $\varepsilon_{1}$ & Upper limit of filling time \\
\hline$Z$ & Compressibility factor & $\varepsilon_{2}$ & Lower limit of SOC \\
\hline$R_{g}$ & Universal gas constant & $c_{2}$ & \\
\hline$S$ & Area $\left(\mathrm{m}^{2}\right)$ & Subscript & \\
\hline C & Throttling coefficient & $c$ & On-board cylinder \\
\hline$\delta$ & Correction factor & or & Orifice \\
\hline$Q_{c}$ & Cooling demand $(\mathrm{J})$ & sto & Cascade storage bank \\
\hline COP & Coefficient of performance & $c v$ & Control volume \\
\hline$\eta_{c}$ & Compressor efficiency & in & Inlet \\
\hline$W_{f}$ & Refrigerator energy consumption $(\mathrm{J})$ & out & Output \\
\hline$W_{c}$ & Compressor energy consumption $(\mathrm{J})$ & sur & Surface of cylinder \\
\hline$W$ & Total energy consumption $(\mathrm{J})$ & wall & Liner inner wall \\
\hline$u$ & Specific internal energy $(\mathrm{kJ} / \mathrm{kg})$ & $a m b$ & Ambient \\
\hline$h$ & Specific enthalpy $(\mathrm{kJ} / \mathrm{kg})$ & pre & Predict \\
\hline$z$ & Height (m) & act & Actual \\
\hline
\end{tabular}

temperature and cylinder dimensions were investigated and shown that hydrogen pre-cooling could effectively reduce the maximum gas temperature. The theoretical model of the refueling process established by Li et al. [21] was applied to research the effect of different geometries and inconstant mass flow rates on temperature rises within cylinders during refueling. An accurate mathematical model can be a convenient, cheap and effective way to research the effect of different parameters during filling process comparing with difficult and costly experiments.

The construction and operation of HRSs requires high investments. Currently, multi-stage filling with hydrogen pre-cooling are being widely used in hydrogen filling stations. The pre-cooling of hydrogen can effectively reduce the temperature rise and multi-stage filling can decrease the energy consumption [22]. Zheng et.al [23] have proposed an optimized control method for cascade HRSs, which could improve the utilization ratio of the cascade storage system while accomplishing fast fillings. The total energy consumption in cascade refueling stations has been studied by Rothuizen et al.[24] and the result shows that the energy consumption is effected by the number and volume of reservoirs and the pressure within reservoirs. The energy saved with a cascade storage has also been estimated as a function of the number of stages in $[25,26]$ it can reach $16.5 \%$ with 3 stages and $20 \%$ with 4 stages refueled to $35 \mathrm{MPa}$. The prediction of temperature of hydrogen flowing from pre-cooler to inlet of vehicle tank could contribute to developing a control strategy of the pre-cooling system [27]. Different filling strategies and effects on key-parameters of the refueling process were researched with CFD model [28]. The most convenient strategy

Reservoir tanks

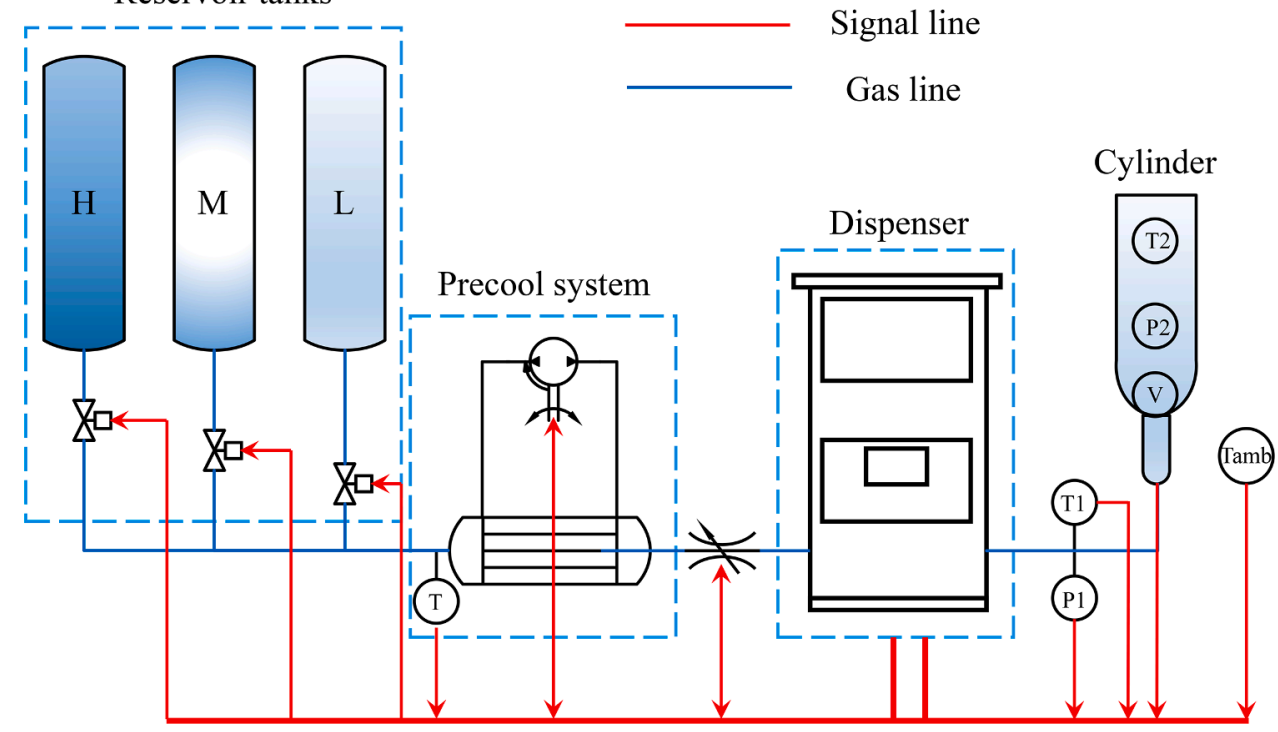

Fig. 1. Diagram of the cascade hydrogen refueling station 
Table 1

Parameter description for the cascade hydrogen refueling station

\begin{tabular}{|c|c|c|c|}
\hline Parameter & Description & Parameter & Description \\
\hline $\mathrm{H}$ & High-pressure reservoir & $\mathrm{T} 2$ & $\begin{array}{l}\text { Temperature within } \\
\text { cylinder }\end{array}$ \\
\hline M & Medium-pressure reservoir & Tamb & Ambient temperature \\
\hline $\mathrm{L}$ & Low-pressure reservoir & P1 & $\begin{array}{l}\text { Pressure of inlet } \\
\text { hydrogen }\end{array}$ \\
\hline $\mathrm{T}$ & $\begin{array}{l}\text { Temperature of cascade } \\
\text { reservoirs }\end{array}$ & P2 & Pressure within cylinder \\
\hline $\mathrm{T} 1$ & $\begin{array}{l}\text { Temperature of inlet } \\
\text { hydrogen }\end{array}$ & $\mathrm{V}$ & Properties of cylinder \\
\hline
\end{tabular}

considering the pre-cooling energy is then identified as starting pre-cooling in the half of the process with a linear pressure rise: a $60 \%$ reduction of the cooling demand is achieved compared to the case of pre-cooling during the whole filling. As seen above, cascade storage system could reduce the energy consumption of HRSs and the pre-cooling may be the most satisfied approach to decrease the temperature rise. Then, a reasonable control strategy for the cascade HRS would increase the filling speed and reduce energy consumption. Therefore, adopting appropriate operational strategies could reduce operating costs and fast filling strategies are very important for HRSs operation, but few researches on the control strategy of cascade HRSs have been conducted.

In this study, the cascade storage hydrogen station is considered and a $35 \mathrm{MPa}$ type III cylinder is investigated. The thermodynamic model is established, which allow calculating the hydrogen pressure and temperature rise within cylinder. According to the thermodynamic model, it is found that the filling time mainly depends on the pressure switching coefficient and the pre-cooling temperature could effectively reduce the temperature rise. Then, a multi-objective optimization model is proposed to calculate the two parameters (pressure switching coefficient, hydrogen pre-cooling temperature ) by considering the energy consumption, the filling time and the SOC of hydrogen cylinder (which are the optimization objectives). Simulation results show that the fast filling process could be accomplished efficiently in different initial conditions. The application of the optimization algorithm on the real-time precooling system control is discussed at the end.

\section{System modelling and setting}

\subsection{Cascade hydrogen refueling stations system}

Cascade storage system is generally used in HRSs considering the hydrogen compression energy consumption. [29-31]. Fig. 1 shows a schematic diagram of a cascade storage system and the parameters in a cascade HRS are illustrated in Table 1.

When the fuel cell vehicle needs to be fed, the cylinder is connected to the dispenser. The level of pressure is switched with the increasing within cylinder and the pre-cooling system could be determined whether to work at the operating temperature during the whole filling process. Filling is finished when the pressure within cylinder reaches the target pressure (higher than $35 \mathrm{MPa}$ as temperature is higher than $288 \mathrm{~K}$ ) or when the temperature on the inner cylinder wall is higher than $358 \mathrm{~K}$.

The hydrogen state of charge (SOC), defined as the mass of charged hydrogen divided by the mass which the cylinder can hold at the condition of $288 \mathrm{~K}, 35 \mathrm{MPa}$, greatly affected the driving range of vehicles. The value of SOC can be calculated by equation (1).

SOC $=\frac{m_{c}(\text { final })}{\rho_{g}(288 K, 35 M P a) V_{c}}$

\subsection{Modelling}

The modeling theory of Compressed Hydrogen Gas (CHG) refueling station and the fast filling process is described in this section. Several assumptions are made in the

model, as follows, to get a simpler set of equations to solve:

- Temperature and pressure within the cascade hydrogen storage system are assumed constant: reservoirs are large with respect to the on-board cylinder, pressure and temperature decrease during the filling of only 1 on-board cylinder are small and neglected.

- ased on the lumped parameters thermodynamic model, temperature and pressure are assumed uniform inside on-board cylinder [32].

- The hydrogen flow is supposed adiabatic within pipes due to the fast speed and short residence time: as shown in [33], even if heat convection is high in the pipes, the heat losses are low and negligible. Pressure drops in these pipes are not taken into account.

- The nozzle of the filling interface is assumed to be a regular hole.

- The transient process is a quasi-steady process. In particular, only the heat accumulation in the $\mathrm{Al}$ liner of the cylinder is taken into account and temperature distribution of the liner is assumed to be uniform.

- Hydrogen is considered as a real gas; but the relation between $\mathrm{u}$ and T uses a simplified form.

- The temperature of hydrogen within cylinder is assumed as the highest temperature of cylinder wall for that the temperature of gas is slightly higher than the temperature of cylinder wall which could ensure the temperature of wall does not exceed the threshold $\left(85^{\circ} \mathrm{C}\right)$.

The detailed theoretical equations carried out to develop the model in this study are presented in the following sections. The following content concerns the models of real gas equation of state, on-board hydrogen cylinder, cascade reservoirs and pre-cooling system.

\subsubsection{State equations of the high-pressure hydrogen}

Generally, low-pressure hydrogen is considered as ideal gas. Nevertheless, hydrogen can no longer be considered as ideal gas under high pressure. In order to simulate the hydrogen state more realistically, the real gas equation with a compression factor $Z_{m}$ is considered as equation (2) [34].

$P V=Z m R_{g} T$

\subsubsection{Hydrogen mass flow rate}

The mass flow rate of hydrogen passing through the valve is calculated based on theoretical formulas for the calculation of the mass flow rate passing through an orifice. The inlet mass flow rate can be calculated by the equation (3)-(6) considering the hydrogen expansion through the orifice is isentropic [9].

$P_{c} / P_{\text {sto }}>\left(\frac{2}{k+1}\right)^{\frac{k}{k-1}}$

$q_{m}=C \rho_{g} S_{o r}\left(\frac{P_{c}}{P_{\text {sto }}}\right)^{\frac{1}{k}}\left\{\left(\frac{2 k}{k-1}\right)\left(\frac{P_{c}}{P_{\text {sto }}}\right)\left[1-\left(\frac{P_{c}}{P_{\text {sto }}}\right)^{\frac{k-1}{k}}\right]\right\}^{\frac{1}{2}}$

$P_{c} / P_{s t o} \leq\left(\frac{2}{k+1}\right)^{\frac{k}{k-1}}$

$q_{m}=C\left(k \rho_{g} P_{s t o}\right)^{\frac{1}{2}} S_{o r}\left(\frac{2}{k+1}\right)^{\frac{k+1}{2(k-1)}}$

The $\mathrm{k}$ is the ratio of specific heats for hydrogen which can be calculate by equation (7).

$k=c_{p} / c_{v}$

Equation (4) and equation (6) are valid for subsonic and sonic conditions of the hydrogen flow respectively. $P_{c}$ and $P_{\text {sto }}$ represent the pressure of cylinder and pressure of cascade storage system. $C$ is the discharging coefficient of the orifice. $\rho_{g}$ is the density of hydrogen and $S_{o r}$ is the area of the valve orifice. 


\subsubsection{Energy and mass balance}

The on-board cylinder is studied as a thermodynamic control volume to find their thermodynamic properties. The mass balance are expressed as equations (8)-(9).

$\frac{d m_{c}}{d t}=\dot{m}$

$m_{\text {in }}=m_{\text {out }}=\dot{m}$

The internal energy of hydrogen is expressed as a function of temperature shown as equation (10). Then, the method to calculate the enthalpy of hydrogen gas is given by equation (11).

$u=a T-b$

$h=u+p / \rho_{g}$

According to the National Institute of Standards and Technology (NIST) data [35], a and b are assumed as constants, 10.51 and 482.43, respectively. Then, $p$ is the pressure of the hydrogen and the density of the hydrogen is expressed by $\rho_{g}$.

In order to calculate the variation of the internal energy within cylinder, the first law of thermodynamic is expressed as a general control volume in equation (12).

$$
\begin{aligned}
\frac{d}{d t}\left[m\left(u+\frac{v^{2}}{2}+g z\right)\right]_{c v}= & \dot{Q}+\sum \dot{m}_{\text {in }}\left(h_{\text {in }}+\frac{v_{\text {in }}^{2}}{2}+g z_{\text {in }}\right) \\
& -\sum \dot{m}_{\text {out }}\left(h_{\text {out }}+\frac{v_{\text {out }}^{2}}{2}+g z_{\text {out }}\right)-\dot{W}_{c v}
\end{aligned}
$$

Because there is no work input and little change in potential energy, the work term $W c v$ is zero and $\mathrm{z}=$ constant. The variation of internal energy within cylinder can be expressed as equation (13).

$\frac{d U_{c}}{d t}=\dot{Q}+m_{i n}\left(\frac{V_{i n}^{2}}{2}+h_{i n}\right)$

As there is no work input between the outlet of the storage and the inlet of the on board cylinder and no heat loss in the pipes and considering here that the pre-cooling system do not extract any heat, there is no change in the hydrogen total enthalpy between the storage outlet and the cylinder inlet. Then, $h_{\text {sto }}=\frac{V_{i n}^{2}}{2}+h_{\text {in }}$, and the energy balance equation can further be simplified as equation (14).

$\frac{d U_{c}}{d t}=\dot{Q}+m_{i n} h_{s t o}$

As only the heat accumulation in the liner is considered, heat transfer between hydrogen and liner is expressed as equation (15). According to previous researches [36-38], the convection heat transfer coefficient is related to the relative velocity of hydrogen and wall, which is reflected as the mass flow rate of the hydrogen and pressure level in this study. Therefore, the convection heat transfer coefficient $k_{1}$ is simply fitted as equation (16).

$Q_{1}=k_{1} S_{\text {wall }}\left(T_{g}-T_{\text {wall }}\right)$

$k_{1}=\delta q_{m}\left(P_{N} / P_{s t o}\right)$

where the $\delta$ is the correction factor for the calculation of $k_{1} . P_{N}$ is the rated pressure of the cylinder (35MPa) and $P_{\text {sto }}$ represents the pressure level filling currently.

The heat transfer from cylinder toward environment is simply expressed as equation (17) [39]. Tamb is also the initial temperature of the cylinder.

$\dot{Q}_{2}=k_{2} S_{\text {sur }}\left(T_{\text {wall }}-T_{\text {amb }}\right)$

where $k_{2}$ is the convective heat transfer coefficient between the wall and ambient, which is assumed as $8 \mathrm{~W} /\left(\mathrm{m}^{2} \bullet \mathrm{K}\right) . S_{\text {sur }}$ is the surface area of the cylinder.

According to the heat absorbed and released of the wall, the temperature of wall is calculated with equation (18)

$c m \Delta T_{\text {wall }}=Q_{1}-Q_{2}$

According to the equations (8)-(15), the total internal energy of hydrogen within cylinder is expressed as equation (19).

$U_{c}=m u+\int q_{m} h_{s t o} d t+\int Q_{1} d t$

In the case where some heat is extracted by the pre-cooling system; $h_{\text {sto }}$ has just to replace by $h_{\text {cooling }}$ in equation (20), which becomes:

$U_{c}=m u+\int q_{m} h_{\text {cooling }} d t+\int Q_{1} d t$

\subsection{Numerical procedure}

At each time step, equation (4) (or (6)) is applied to evaluate the inlet mass flow. Equation (8) and (9) are used to calculate the in-cylinder mass with the mass flow rate above. Next, equation (19) is utilized to calculate internal energy of hydrogen within cylinder at each time step. According to the mass and internal energy of hydrogen within cylinder, the other properties (pressure and temperature) of hydrogen can be found with equations (10), (11) and the state equation (2) of hydrogen for the on-board cylinder. The pressure source of cascade storage system needs to be switched during the procedure process. The procedure continues until the pressure within cylinder reaches the targeted pressure.

\section{Optimization method}

In this section, the determination of pressure switching coefficients (PSC) and the calculation of cooling and compression demand of the fast filling process are presented. Then, a multi-objective optimization model is build and the optimization algorithm is given.

\subsection{Pressure switching coefficients}

The compressed hydrogen is stored in cascade storage system. The pressure source (i.e. the connected reservoir) must be switched to a higher level when the pressure within cylinder reaches the preset switching pressure $\left(P_{\text {swit }}\right)$ during the filling process. At present, the cascade HRSs are operated by fixed pressure switching points or switch when the pressure within cylinder equals the pressure source. These methods may lead to uncontrolled refilling for different initial conditions and long filling time. In this study, the pressure switching point is set to be a variable which depends on the initial conditions. Hence, setting an appropriate pressure switch point could effectively shorten filling time and affect the filling process according to equation (4) (or (6)). The pressure switching coefficients (PSC) is proposed for a better control of pressure switching which is defined as the ratio of the switching pressure $\left(P_{\text {swit }}\right)$ to the currently filling pressure level $\left(P_{\text {sto }}\right)$. The $P S C$ is a significant parameter which can determine the refueling time and it is considered as an optimization parameter to achieve fast refilling. The effects of the pressure switching point on the filling time and temperature rise are presented in section 4.2.

$P S C=P_{\text {swit }} / P_{\text {sto }}$

Similarly, the hydrogen utilization ratio (HUR) is also defined as the ratio of $P_{\text {swit }}$ to $P_{\text {sto }}$ expressed in equation (22). The smaller $H U R$, (i.e. the lower PSC), the lower is the hydrogen utilization from the low-pressure and medium-pressure reservoirs. Therefore, reasonable PSC can improve HUR of the cascade storage system.

$H U R=P_{\text {swit }} / P_{\text {sto }}$ 


\subsection{Energy consumption}

To reduce the temperature rise within hydrogen cylinder, the hydrogen needs to be pre-cooled with a refrigerator facility before the inlet into the cylinder. The cooling demand of the refrigerator facility is calculated in the model with the energy balance in the heat exchanger as equation (23).

$Q_{c}=\Delta h \dot{m}$

$\Delta h=h_{\text {sto }}-h_{\text {cooling }}$

The energy consumption $W$ of refrigeration facility is expressed as equation (25).

$W_{f}=Q_{c} /$
$C O P$

where $C O P$ is the coefficient of performance for the refrigeration facility. The energy consumption mainly depends on the pre-cooling temperature which could reduce the temperature rise effectively.

The energy consumption of compressor with different gas feeding methods is different. The more gas is taken from the low pressure cylinder group, the lower energy consumption will be. On the contrary, taking gas from a high-pressure cylinders may need more compression energy. Considering the compression demand, selecting a reasonable gas feeding method could reduce the energy consumption of hydrogen compression. Assuming hydrogen is compressed with isentropic, the temperature of hydrogen outflowed from compressor could be calculated by equation (26). Then, the energy consumption of the compressor can be calculated with equation (27), according to the conservation of energy and the formula of hydrogen enthalpy, as for equation (10) and (11).

$T_{\text {out }}=T_{\text {in }}\left(P_{\text {out }} / P_{\text {in }}\right)((k-1) / k)$

$W_{c}=\left(h_{\text {out }}-h_{\text {in }}\right) \eta_{c}$

where $T_{\text {in }}, P_{\text {in }}, T_{\text {in }}, T_{\text {out }} h_{\text {in }}, h_{\text {out }}$ are the temperatures, pressures and enthalpies of hydrogen flowing in and out of respectively. $W_{c}$ is the compression energy and $\eta_{c}$ represents the compressor efficiency.

The cooling demand and compression energy are considered as the total energy consumption as equation (28).

$W=W_{f}+W_{c}$

\subsection{Optimization algorithm}

The cascade refueling process has been described and the simulation model established in the previous section. Based on the above analysis, the energy consumption, filling time and SOC need to be quantified with the PSC and pre-cooling temperature in a certain initial condition. Therefore, the total energy consumption, filling time and SOC are considered as optimization objectives. The PSC and pre-cooling temperature are taken as the optimization parameters (x). The mathematical model is presented in equation (29) and (30).

$\min [W(x), t(x),-S O C(x)]$

s.t. $x \in X$

where the $W(x)$, energy consumption is set as the main target. $t(x)$ is the filling time constraint. $\operatorname{SOC}(x)$ represents the mass of hydrogen within cylinder.

To simplify the optimization model, the $t(x)$ and $\operatorname{SOC}(x)$ could be set as constraint conditions. The limitation of filling time is $180 \mathrm{~s}$ (3min). According to the international standard of SAE-J2601, the target SOC is required higher than $95 \%$ when fueling with communications. However,

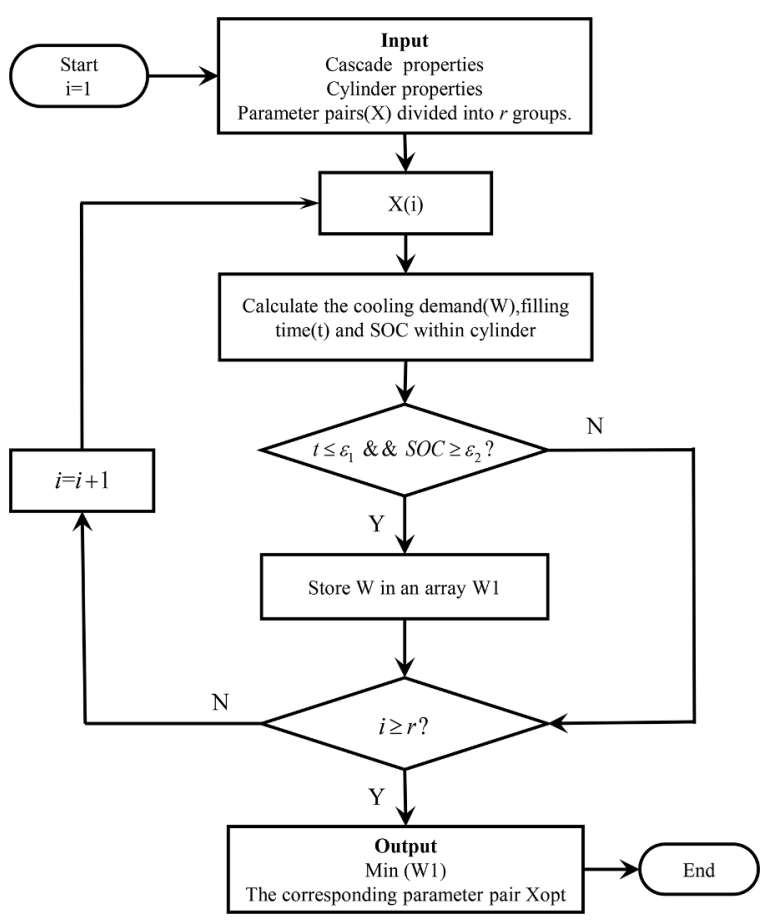

Fig. 2. Flow chart of the optimization algorithm

the pressure inside the cylinder after refilling is greater than $35 \mathrm{MPa}$ in SAE-J2601. In this paper, the pressure inside the cylinder do not exceed the rated pressure of $35 \mathrm{MPa}$ to ensure the operation safety and service life of the cylinder. In addition, in order to accomplish fast refueling and ensure the safety during refueling process, which is under an extremely harsh working conditions (high ambient temperature and low initial pressure within cylinder), thus, the lower limit SOC was set as $85 \%$. The content of the $\mathrm{X}$ domain refers to the data range of $P S C$ and pre-cooling temperature. Considering the hydrogen utilization ratio and filling time of cascade storage system, the PSC varies from 0.55 to 0.95 . Besides, the temperature of inlet hydrogen could be pre-cooled from ambient temperature to $-40^{\circ} \mathrm{C}$. The single-objective optimization model is shown as equation (31) and (32).

$\min W(x)$

s.t. $\left\{\begin{array}{l}t(x) \leq \varepsilon_{1} \\ \operatorname{SOC}(x) \geq \varepsilon_{2} \\ x \in X\end{array}\right.$

The mathematical model above could be solved with the sequence search algorithm shown in Fig. 2. When the on-board hydrogen system is connected to HRS, the cylinder properties are transmitted to the dispenser through infrared communication. The parameters pairs (X (i)) is divided into $\mathrm{r}$ groups, for example, $\mathrm{X}(1)=\left[0.55,-40^{\circ} \mathrm{C}\right]$. With the properties of cascade system, PSC and pre-cooling temperature are given, the energy consumption, filling time and SOC could be calculated with the currently initial condition. Parameters trade-offs are made by comparing the filling time and SOC with the constraint conditions. Then, all the parameter pairs that satisfy the constraints are recorded and the one with the lowest energy consumption is the optimal solution. This procedure continues with different initial conditions.

\section{Result and Discussion}

In this study, the pressure level of the cascade storage system is set as high, medium and low pressure (40, 30 and $20 \mathrm{MPa}$ respectively) according to the operation parameters of a hydrogen station. Then, a type III cylinder was selected for the investigation with a nominal pressure of 
Table 2

Physical characteristics of the tested Type III cylinder

\begin{tabular}{llllll}
\hline Characteristics & $\begin{array}{l}\text { Nominal } \\
\text { pressure } \\
(\mathrm{MPa})\end{array}$ & $\begin{array}{l}\text { Storage } \\
\text { volume } \\
(\mathrm{L})\end{array}$ & $\begin{array}{l}\text { Length } \\
(\mathrm{m})\end{array}$ & $\begin{array}{l}\text { Inner } \\
\text { diameter } \\
(\mathrm{m})\end{array}$ & $\begin{array}{l}\text { External } \\
\text { diameter } \\
(\mathrm{m})\end{array}$ \\
Value & 35 & 140 & 1.78 & 0.334 & 0.374 \\
\hline
\end{tabular}

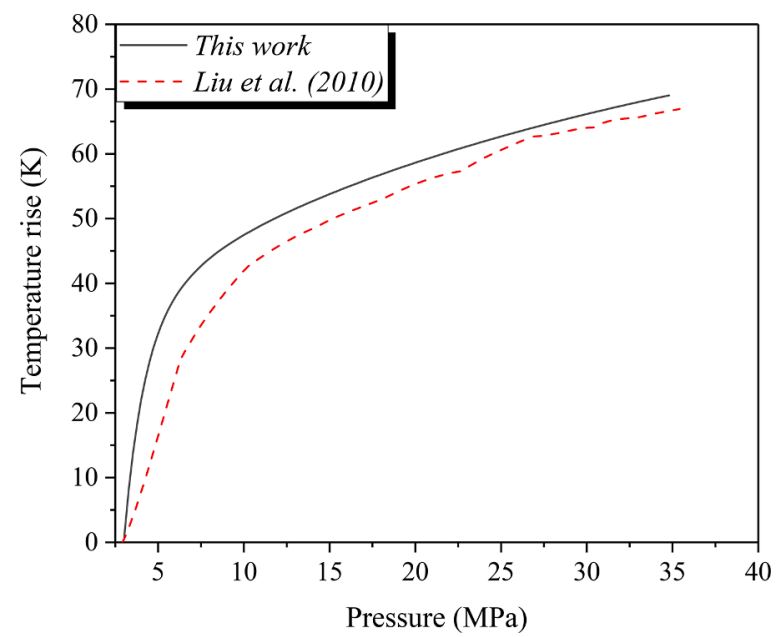

Fig. 3. Comparison of experimental results [15] and simulation results for temperature rise vs. pressure increase

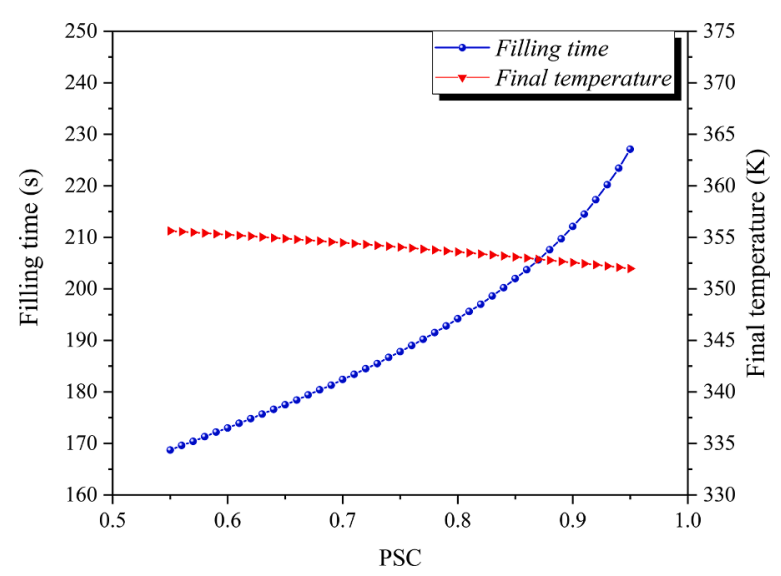

Fig. 4. The variation of filling time and final temperature with PSCs

$35 \mathrm{MPa}$, volume of $140 \mathrm{~L}$ and orifice diameter of $7.5 \mathrm{~mm}$, respectively. The physical characteristics of the tested Type III cylinder are presented as Table 2. The effects of PSC and pre-cooling temperature for the filling process are investigated after the model validation. Besides, the results of the multi-objective optimization model under different initial conditions are presented.

\subsection{Model validation}

The model was validated with the experimental data carried out with a 35MPa, 150L, type III cylinder by Liu et al. [15] The initial temperature and pressure are set as $293 \mathrm{~K}$ and $3 \mathrm{MPa}$ respectively. In Fig. 3, the comparison of experimental and simulation results is presented. It can be seen that simulation results have the same variation trend as the experimental data and are in good agreement (with less than $3 \mathrm{~K}$ of temperature rise at the end of the filling). The general difference of temperature rise may be caused by the model assumptions. Therefore, the model is relatively accurate and can be used for the further research.

\subsection{Effect of PSC and pre-cooling temperature}

For the cascade HRSs, PSC and pre-cooling temperature are two controllable variables which may affect the filling process significantly according to the thermodynamic model. Hence, the optimization model proposed in this paper mainly studies the optimization of PSC and precooling temperature. The filling strategy could be optimized by studying the effects of the above two parameters on the filling process.

With an initial condition of ambient temperature $293 \mathrm{~K}$, initial pressure $2 \mathrm{MPa}$ and inlet hydrogen pre-cooled to $273 \mathrm{~K}$, the results calculated with different pressure switching points are shown in Fig. 4. The filling time is rising greatly with the increasing of the PSC and the variation of the refueling time is about $2 \mathrm{~s}$ with a switch of 0.01 for the $P S C$. At the same time, the final temperature within the cylinder varies less than $5^{\circ} \mathrm{C}$ in the full range of $P S C$ level. In consequence, $P S C$ level is the main influence factor of filling time. This behavior can be explained by equation (4) (or (6)). According to the above analysis, setting a reasonable PSC can effectively decrease the filling time and has little effect on the final temperature within the cylinder. But one should keep in mind that decreasing the PSC reduces the HUR.

With an initial condition of ambient temperature $293 \mathrm{~K}$, initial pressure $2 \mathrm{MPa}$ and PSC 0.65 , the variation of filling time and final temperature within cylinder was shown as Fig. 5 with different inlet hydrogen temperature. It is obvious that the filling time changes little as hydrogen pre-cooling temperature varies. On the contrary, the relationship between the final temperature and pre-cooling temperature is approximately linear and the final temperature within the cylinder has a marked change with the pre-cooling temperature. This is due to the internal energy of the inlet hydrogen which is greatly reduced through pre-cooling. As can be seen, for a $2{ }^{\circ} \mathrm{C}$ reduction in hydrogen pre-cooling temperature, the final temperature within the cylinder decreases approximately $1^{\circ} \mathrm{C}$, which could provide the idea for the determination of the necessary pre-cooling temperature.

\subsection{Optimization with different initial conditions}

The energy consumptions with different optimization parameters under initial pressure $2 \mathrm{MPa}$ and ambient temperature $293 \mathrm{~K}$ are presented in Fig. 6 (a). The points that do not satisfy the constraints (filling time 3min and SOC 85\%) have been omitted. Energy consumption increases sharply with the decrease of pre-cooling temperature due to the change in enthalpy of hydrogen. On the contrary, energy consumption increases slightly with the increase of PSC on account of more hydrogen is fed into the on-board cylinder. As we can see, the energy consumption reaches minimum with the PSC 0.61 and pre-cooling temperature $261 \mathrm{~K}$. Filling time and SOC within cylinder with different optimization

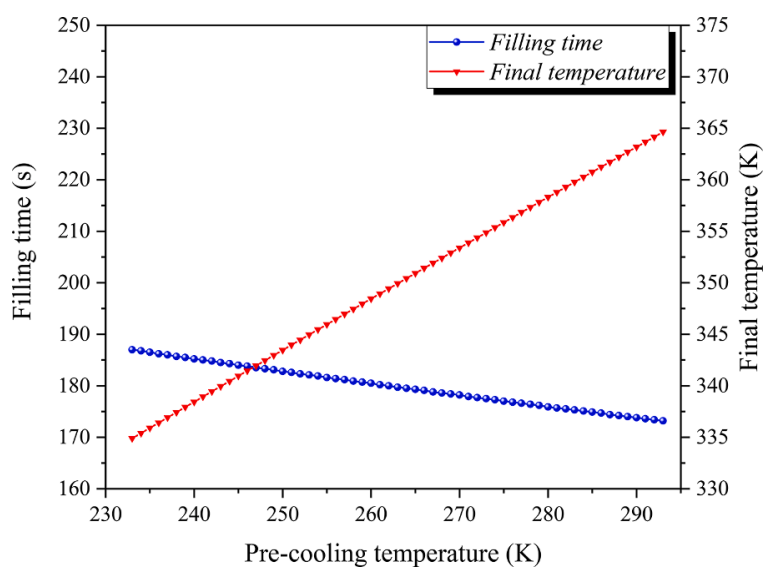

Fig. 5. The variation of filling time and final temperature with pre-cooling temperature 

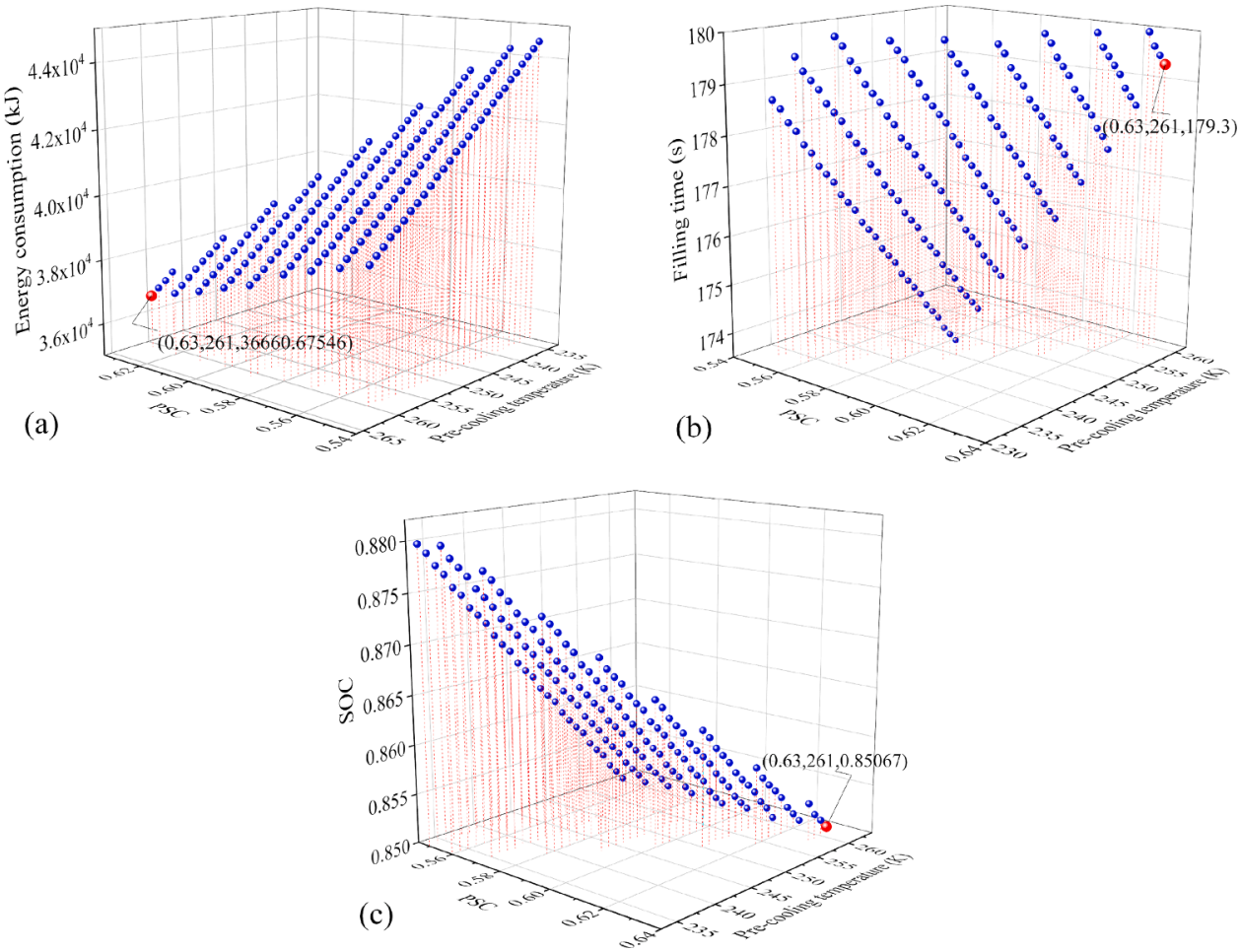

Fig. 6. Optimized result under initial pressure $2 \mathrm{MPa}$ and ambient temperature $293 \mathrm{~K}$. (a) Energy consumption under different parameters. (b) SOC with different parameters. (c) Filling time under different parameters.

Table 3

Optimized parameters and target under initial condition 2MPa, 293K

\begin{tabular}{|c|c|c|c|c|c|c|}
\hline Parameter & PSC & Pre-cooling temperature (K) & Energy consumption (kJ) & Energy of hydrogen fed $(\mathrm{kJ})$ & Filling time (s) & SOC \\
\hline
\end{tabular}

parameters are presented in Fig. 6 (b) and (c). The filling times are all less than $180 \mathrm{~s}$ ( $3 \mathrm{~min}$ ) and the SOC are all higher than $85 \%$. Considering hydrogen calorific value as $1.4 * 10^{\wedge} 5 \mathrm{~kJ} / \mathrm{kg}$, the energy of hydrogen fed into cylinder is also calculated to compare with the energy consumption. The detailed optimized parameters and targets are shown in Table 3.

The energy distribution of precooling and compression energy consumption is shown in Fig. 7 for a refueling process. Fig. 7 (a) shows the actual energy consumption during fast filling process. We can see that large PSC can reduce compression energy consumption and improve hydrogen utilization. In the meanwhile, pre-cooling energy decreases with the increase of precooling temperature. Therefore, the total energy consumption can be minimized by parameter optimization. The ratio of the energy consumption between components is shown in Fig. 7 (b). The compressor accounts for more than $80 \%$ and the refrigeration facility only account for a small part.

Considering the actual operating conditions of HRSs, the ambient temperature (and initial on-board cylinder temperature) varies from 273 $\mathrm{K}$ to $313 \mathrm{~K}$ with a step of $1 \mathrm{~K}$ and initial pressure within cylinder varies from $2 \mathrm{MPa}$ to $15 \mathrm{MPa}$ with a step of $1 \mathrm{MPa}$. Herein, there are 574 different initial conditions calculated by parameter combination and the optimized results are presented as Fig. 8.

The optimal parameter of PSC is presented in Fig. 8 (a). A small PSC
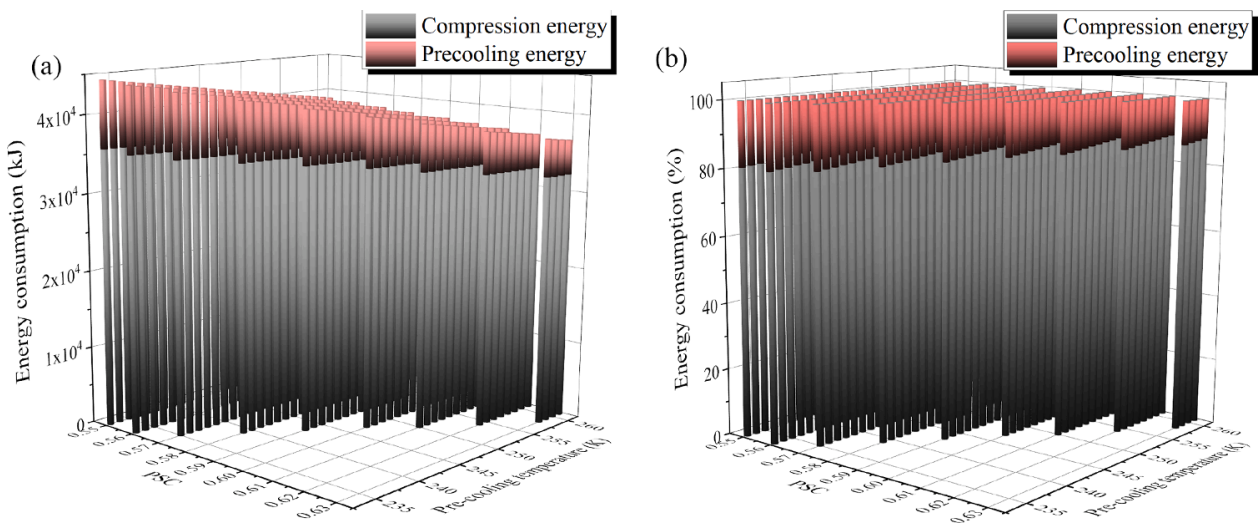

Fig. 7. Energy distribution between the compressor and the cooling facilities under initial pressure $2 \mathrm{MPa}$ and ambient temperature $293 \mathrm{~K}$. (a) Actual energy consumption. (b) Energy consumption ratio. 

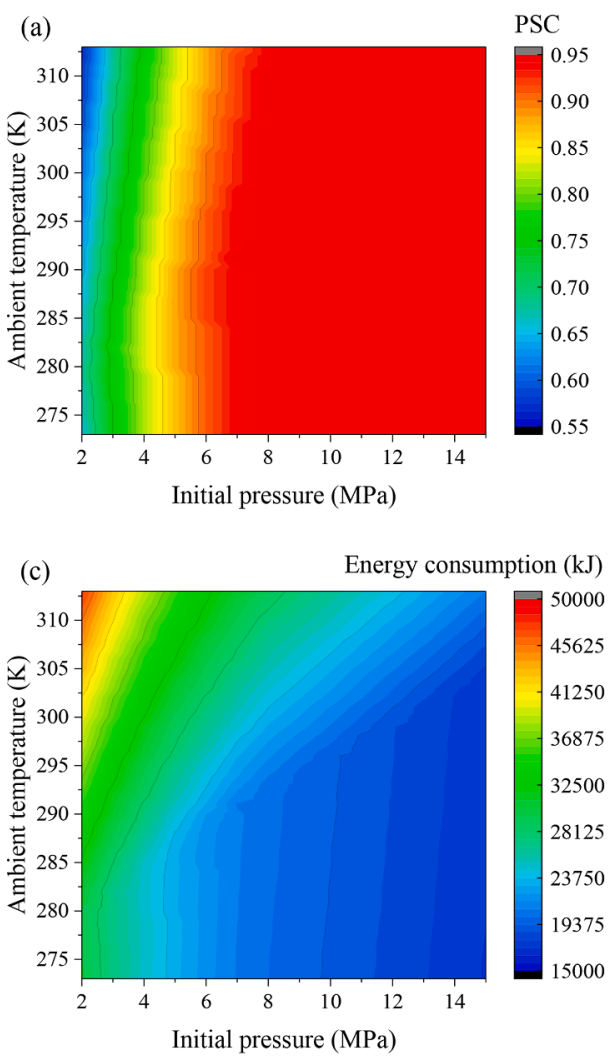
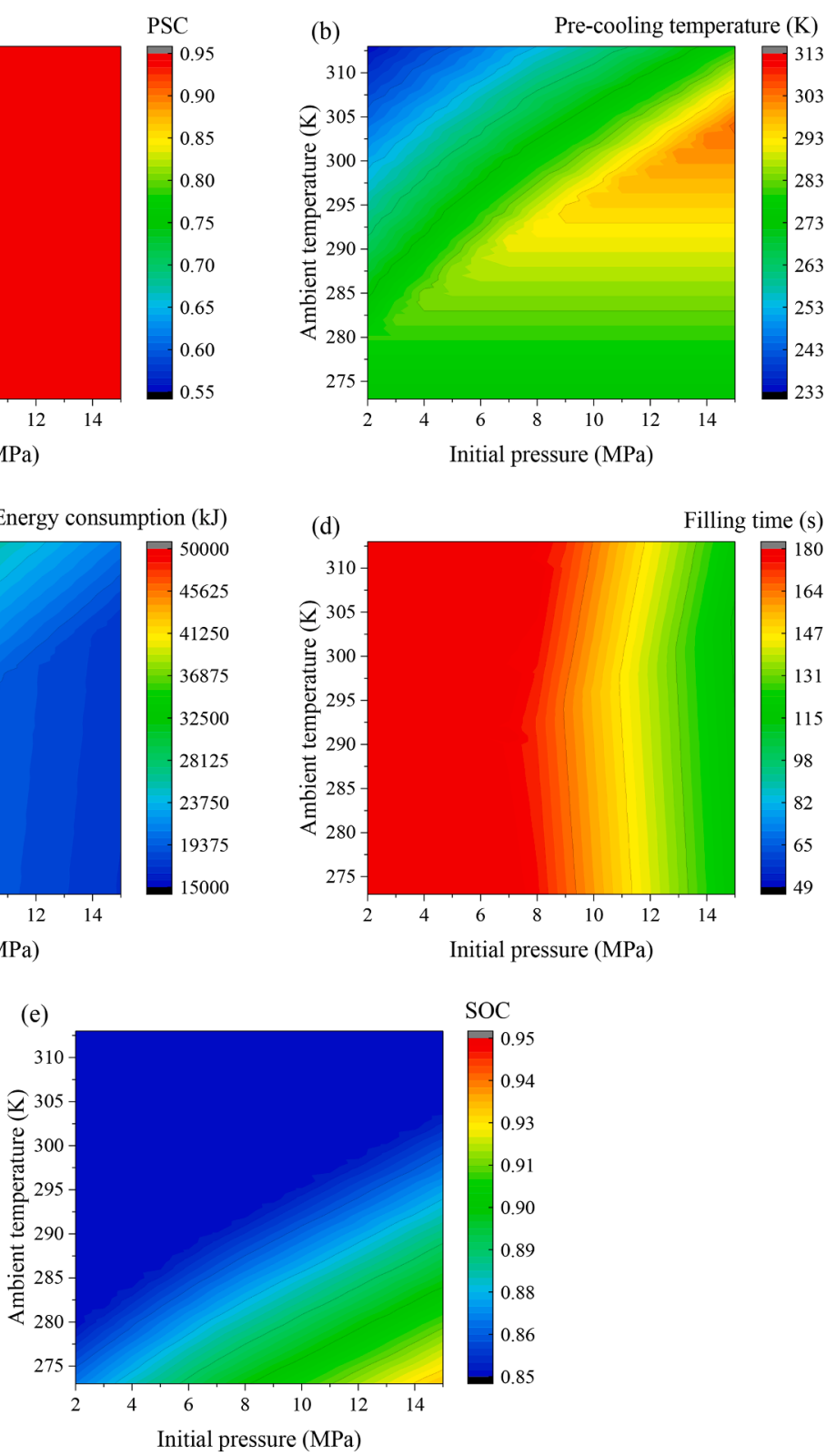

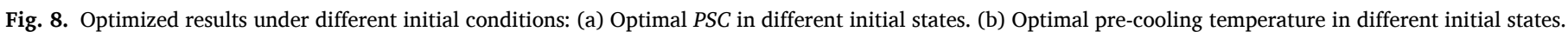
(c) The minimum energy consumption under different initial states. (d) and (e) The optimized filling time and SOC in different initial states.

should be selected to complete the filling process within 3 min when the initial pressure and the ambient temperature are low. Then, at the given temperature, when initial pressure increases, PSC can be increased. But then $P S C$ has to be decreases gradually when the initial pressure is high. This is because when the ambient temperature is high, a larger PSC can slightly reduce the temperature rise, compression energy and improve SOC meanwhile. When the initial pressure is high, SOC constraint can easily be met and large PSC could decrease compression energy consumption effectively.

The optimal pre-cooling temperature of inlet hydrogen is given in Fig. 8 (b). The pre-cooling temperature decreases with the high ambient temperature and low initial pressure because these two conditions lead to a large temperature rise. When the ambient temperature is low and the initial pressure is high, the inlet hydrogen requires less pre-cooling or even no pre-cooling.

Fig. 8. (c) presents the minimum energy consumption under different initial conditions. The energy consumption of pre-cooling reaches a maximum when the ambient temperature is the highest and the initial pressure is the lowest. Because these working conditions result in a sharp temperature rise and require very low pre-cooling temperature.

The filling time with the optimal parameters is given in Fig. 8 (d). As we can see, the filling time decreases with the increase of initial pressure because long filling time means a large PSC which could decrease compression energy. The ambient temperature has little effect on filling time.

Fig. 8 (e) presents the SOC with different initial states. The results indicate that SOC changes are inversely proportional to the ambient temperature and directly proportional to the initial pressure. To reduce energy consumption, SOC is controlled within lower limit in these extreme cases of high ambient temperature and low initial pressure.

\subsection{Application on cascade hydrogen station control}

A high temperature rise may occur in the on-board cylinder due to the fast filling speed in a cascade HRSs. Therefore, an optimized control strategy should effectively control the filling process. At present, the 


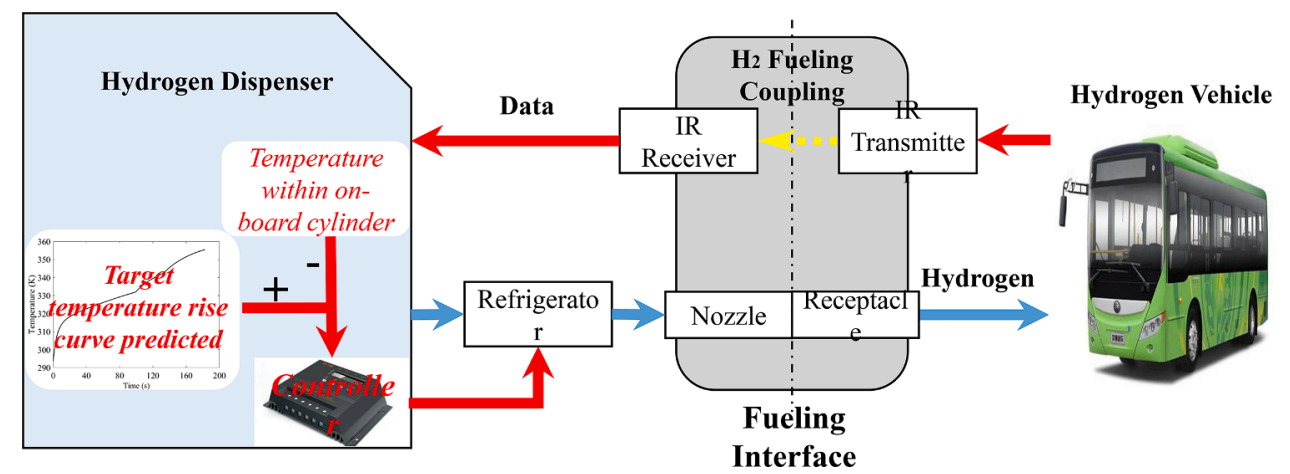

Fig. 9. Implementation process of real-time control for cascade HRS [40]

cascade hydrogen stations are operated with fixed pressure switching points and fixed pre-cooling temperatures, which leads to either longer filling times or higher energy consumptions. Herein, in order to overcome the drawback of cascade HRSs, a real-time control method of the pre-cooling system is presented and discussed. Fig. 9 shows the implementation process of the proposed control method for communicating HRSs.

With the optimal algorithm proposed in section III, the optimization parameters of $P S C$ and pre-cooling temperature could be calculated in advance with lookup tables like in SAE-J2601 or calculated in real-time according to the initial parameters. Then, the model can calculate the temperature-rise curve of the filling process as the target curve, based on these two parameters. In general, the filling process could be controlled with the optimal PSC and pre-cooling temperature calculated above. However, due to the hysteresis of control, the variable environment factors and the approximations of the model, the actual temperature rise during the filling process may be not exactly as the model predicted. Therefore, the refrigerator could be controlled by feedback to ensure the actual temperature rise within the cylinder is as close as possible to the predicted and acceptable curve during the filling process. This control method could provide a new effective control for cascade HRSs.

\section{Conclusion}

In this study, an iterative lumped parameter thermodynamic model for the fast filling process of on-board cylinders is developed considering the real gas equation of state, energy and mass balance. Considering the energy consumption and operation cost of the HRSs, the cascade hydrogen station is considered. The effects of PSC and pre-cooling temperature during the filling process are studied. An optimized filling algorithm is proposed to achieve low energy consumption, fast filling and high SOC within on-board cylinders. The controllable variables, PSC and pre-cooling temperature, are selected as optimization parameters. Optimization results under different initial conditions are given. The results show that the optimization algorithm could achieve the lowest energy consumption with shortest filling time and highest SOC. The application of the optimization algorithm on the real-time pre-cooling system control is discussed.

\section{Author Statement}

\section{Declaration of Competing Interest}

The authors declared that they have no conflicts of interest to this work. We declare that we do not have any commercial or associative interest that represents a conflict of interest in connection with the work submitted.

\section{Acknowledgments}

This work is supported in part by the national key research and development program (No.: 2018YFB0105703 and No.: 2018YFB0105402), Graduate scientific research and innovation foundation of Chongqing, China (No.: CYS19020).

\section{References}

[1] D Cecere, E Giacomazzi, A. Ingenito, A review on hydrogen industrial aerospace applications, International Journal of Hydrogen Energy 39 (2014) 10731-10747.

[2] Suman. Dutta, A review on production, storage of hydrogen and its utilization as an energy resource, Journal of Industrial Engineering Chemistry 20 (2014) $1148-1156$.

[3] M Gurz, E Baltacioglu, Y Hames, K. Kaya, The meeting of hydrogen and automotive: A review, International Journal of Hydrogen Energy 42 (2017) 23334-23346.

[4] WRW Daud, RE Rosli, EH Majlan, SAA Hamid, R Mohamed, T. Husaini, PEM fuel cell system control: A review. Renewable Energy. 113 (2017) 620-638.

[5] G Fabian, D Lucy, R Martin, S. Detlef, Carsharing with fuel cell vehicles: Sizing hydrogen refueling stations based on refueling behavior, Applied Energy 228 (2018) 1540-1549.

[6] Weber Barthelemy, France F., Barbier. Hydrogen storage: Recent improvements and industrial perspectives, International Journal of Hydrogen Energy (2016) 42.

[7] J Xiao, J Cheng, X Wang, P Benard, R. Chahine, Final hydrogen temperature and mass estimated from refueling parameters, International journal of hydrogen energy 43 (2018) 22409-22418.

[8] J Zheng, X Liu, X Ping, P Liu, Y Zhao, Y. Jian, Development of high pressure gaseous hydrogen storage technologies, International Journal of Hydrogen Energy 37 (2012) 1048-1057.

[9] M Farzaneh-Gord, M Deymi-Dashtebayaz, HR Rahbari, H. Nxazmand, Effects of storage types and conditions on compressed hydrogen fuelling stations performance, International Journal of Hydrogen Energy 37 (2012) p.3500-p.3509.

[10] E Talpacci, M Reuss, T Grube, P Cilibrizzi, R Gunnella, M Robinius, et al., Effect of cascade storage system topology on the cooling energy consumption in fueling stations for hydrogen vehicles, International Journal of Hydrogen Energy 43 (2018) 6256-6265.

[11] Y Wang, J Kowal, M Leuthold, DU. Sauer, Storage System of Renewable Energy Generated Hydrogen for Chemical Industry, World Hydrogen Energy Conference 29 (2012) 657-667.

[12] E Rothuizen, W Merida, M Rokni, M. Wistqft-Ibsen, Optimization of hydrogen vehicle refueling via dynamic simulation, International Journal of Hydrogen Energy 38 (2013) 4221-4231.

[13] S. Engineers, Fueling protocols for light duty gaseous hydrogen surface vehicles, SAE international, 2016.

[14] J Zheng, J Guo, J Yang, Y Zhao, Z Lei, X Pan, et al., Experimental and numerical study on temperature rise within a $70 \mathrm{MPa}$ type III cylinder during fast refueling, International Journal of Hydrogen Energy 38 (2013) 10956-10962.

[15] YL Liu, YZ Zhao, L Zhao, X Li, HG Chen, LF Zhang, et al., Experimental studies on temperature rise within a hydrogen cylinder during refueling, International Journal of Hydrogen Energy 35 (2010) 2627-2632.

[16] MW Dicken C J B, Measured effects of filling time and initial mass on the temperature distribution within a hydrogen cylinder during refuelling, Journal of Power Sources 165 (2007) 324-336.

[17] D Melideo, D Baraldi, NDM Echevarria, BA. Iborra, Effects of some key-parameters on the thermal stratification in hydrogen tanks during the filling process, International journal of hydrogen energy 44 (2019) 13569-13582.

[18] L Zhao, Y Liu, J. Yang, Numerical simulation of temperature rise within hydrogen vehicle cylinder during refueling, International Journal of Hydrogen Energy 35 (2010) 8092-8100.

[19] J Xiao, P Benard, R. Chahine, Estimation of final hydrogen temperature from refueling parameters, International Journal of Hydrogen Energy 42 (2017) $7521-7528$.

[20] M Li, Y Bai, C Zhang, Y Song, S Jiang, D Grouset, et al., Review on the research of hydrogen storage system fast refueling in fuel cell vehicle, International Journal of Hydrogen Energy 44 (2019) 10677-10693. 
[21] Q Li, J Zhou, Q. Chang, Effects of geometry and inconstant mass flow rate on temperatures within a pressurized hydrogen cylinder during refueling, International journal of hydrogen energy 37 (2012) 6043-6052.

[22] J Xiao, X Wang, P. Bénard, Determining hydrogen pre-cooling temperature from refueling parameters, international journal of hydrogen energy 41 (2016) 16316-16321.

[23] J Zheng, J Ye, Y Jian, T Ping, Z Lei, M. Kern, An optimized control method for a high utilization ratio and fast filling speed in hydrogen refueling stations, International Journal of Hydrogen Energy 35 (2010) 3011-3017.

[24] RM Rothuizen E, Optimization of the overall energy consumption in cascade fueling stations for hydrogen vehicles, International Journal of Hydrogen Energy 39 (2014) 582-592.

[25] D Grouset, C Ridart, M. Milhé, in: Lowering energy and costs for hydrogen transportation and distribution. Hypothesis XII symposium, Syracuse, 2017.

[26] D Grouset, C. Ridart, Lowering energy spending together with compression, storage and transportation costs forhydrogen distribution in the early market. In "Design, deployment and operation of a hydrogen supply chain", Coord. Catherine AzzarroPantel, Elsevier ed, 2018.

[27] T Kuroki, N Sakoda, K Shinzato, M Monde, Y. Takata, Prediction of transien temperature of hydrogen flowing from pre-cooler of refueling station to inlet of vehicle tank, International Journal of Hydrogen Energy (2017), S0360319917343239.

[28] D Melideo, D. Baraldi, CFD analysis of fast filling strategies for hydrogen tanks and their effects on key-parameters, International Journal of Hydrogen Energy 40 (2015) 735-745.

[29] M Sadi, M. Deymi-Dashtebayaz, Hydrogen refueling process from the buffer and the cascade storage banks to HV cylinder, International journal of hydrogen energy 44 (2019) 18496-18504.
[30] M D-DM Farzaneh-Gord, H R Rahbari, Studying effects of storage types on performance of CNG filling stations, Journal of Natural Gas Science and Engineering 3 (2011) 334-340.

[31] Morteza Saadat-Targhi, Javad Khadem, Mahmood Farzaneh-Gord, Mathematical modeling of fast filling process at CNG refueling stations considering connecting pipes, Journal of Natural Gas Science Engineering (2015).

[32] M Monde, P Woodfield, T Takano, M. Kosaka, Estimation of temperature change in practical hydrogen pressure tanks being filled at high pressures of 35 and $70 \mathrm{MPa}$, International Journal of Hydrogen Energy 37 (2012) p.5723-p.5734.

[33] D Grouset, C. Ridart, Temperature evolutions during high pressure hydrogen tank refuelling: modelling and time scale analysis, Hypothesis XIII symposium (2017).

[34] EW Lemmon, ML Huber, JW. Leachman, Revised Standardized Equation for Hydrogen Gas Densities for Fuel Consumption Applications 113 (2008) 341-350. Journal of Research of the National Institute of Standards Technology.

[35] National Institute of Standards and Technology. http://webbook.nist.gov/ch emistry/.

[36] WP Monde M, T Takano, Estimation of temperature change in practical hydrogen pressure tanks being filled at high pressures of 35 and $70 \mathrm{MPa}$, International journal of hydrogen energy 37 (2012) 5723-5734.

[37] Bourgeois Thomas, Ammouri Fouad, Weber Mathilde, et al., Evaluating the temperature inside a tank during a filling with highly-pressurized gas, International Journal of Hydrogen Energy 40 (2015) 11748-11755.

[38] PL Woodfield, M Monde, Y. Mitsutake, Measurement of Averaged Heat Transfer Coefficients in High-Pressure Vessel during Charging with Hydrogen, Nitrogen or Argon Gas, Journal of Thermal Science and Technology 2 (2007) 180-191.

[39] V Molkov, M Dadashzadeh, D. Makarov, Physical model of onboard hydrogen storage tank thermal behaviour during fuelling, International Journal of Hydrogen Energy 44 (2019) 4374-4384.

[40] S. Engineers, Hydrogen Surface Vehicle to Station Communications Hardware and Software, SAE international, 2014. 